From Emory University, Atlanta, GA National Cancer Institute, Bethesda; Cancer Therapy Evaluation Program, National Cancer Institute, Rockville, MD; Cancer Therapy and Research Center at The University of Texas Health Science Center, San Antonio, TX: City of Hope Cancer Center, Duarte, CA; Karmanos Cancer Institute. Detroit, Ml; University of Pittsburgh Cancer Institute, Pittsburgh; and Penn State Hershey Cancer Institute, Hershey, PA.

Submitted May 13, 2010; accepted August 2, 2010; published online ahead of print at www.jco.org on September 13, 2010.

Supported by National Institutes of Health Grants No. NO1 CM-62208 (Southeast Phase 2 Consortium); N01-CO-124001, subcontract 25XS115-Task Order 2, U01CA099168, P30CA47904, and Nationa Institutes of Health/General Clinical Research Center 5M01 RR 00056 (University of Pittsburgh); U01 CA-062505 (California Cancer Consortium); and UO1 CA-062487 (Karmanos Cancer Institute)

Presented in part at the 46th Annual Meeting of the American Society of Clinical Oncology, June 4-8, 2010

Chicago, IL.

Authors' disclosures of potential conflicts of interest and author contributions are found at the end of this article.

Clinical Trials repository link available on JCO.org.

Corresponding author: Suresh S. Ramalingam, MD, Emory University, Winship Cancer Institute, 1365 Clifton Rd NE, Room C-3090, Atlanta, GA 30322 e-mail: Suresh.ramalingam@emory.edu.

(C) 2010 by American Society of Clinical Oncology

0732-183X/10/2829-4507/\$20.00

DOI: $10.1200 / \mathrm{JCO} .2010 .30 .2307$

\title{
Phase I Study of Vorinostat in Patients With Advanced Solid Tumors and Hepatic Dysfunction: A National Cancer Institute Organ Dysfunction Working Group Study
}

Suresh S. Ramalingam, Shivaani Kummar, John Sarantopoulos, Stephen Shibata, Patricia LoRusso, Mara Yerk, Julianne Holleran, Yan Lin, Jan H. Beumer, R. Donald Harvey, S. Percy Ivy, Chandra P. Belani, and Merrill J. Egorin

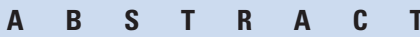

\section{Purpose}

Vorinostat is the first US Food and Drug Administration-approved histone deacetylase inhibitor and is indicated for the treatment of refractory cutaneous T-cell lymphoma. We conducted a phase I study to determine the maximum-tolerated dose and pharmacokinetics of vorinostat in patients with hepatic dysfunction.

\section{Patients and Methods}

Patients had solid malignancies and acceptable bone marrow and renal function. Hepatic dysfunction was categorized as mild, moderate, or severe by the National Cancer Institute Organ Dysfunction Working Group criteria. Fifteen patients with normal liver function were enrolled as controls. All patients received a single 400-mg dose of vorinostat for pharmacokinetic studies. One week later, daily vorinostat dosing was begun and continued until toxicity or disease progression occurred. The daily vorinostat dose was escalated within each hepatic dysfunction category. Vorinostat plasma concentrations were quantitated by a validated liquid chromatography-tandem mass spectrometry assay and modeled noncompartmentally.

\section{Results}

Fifty-seven patients were enrolled (median age, 59 years; females, $\mathrm{n}=24$ ); 42 patients had hepatic dysfunction (16 mild, 15 moderate, and 11 severe). Eight of nine patients with doselimiting toxicity had grade 4 thrombocytopenia. The recommended vorinostat doses in mild, moderate, and severe hepatic dysfunction were 300,200, and $100 \mathrm{mg}$, respectively, on the daily continuous schedule. There were no significant differences in vorinostat pharmacokinetic parameters among the normal or hepatic dysfunction categories. Disease stabilization was noted in 12 patients. Of five patients with adenoid cystic carcinoma, one patient had a partial response, and four patients had stable disease. A patient with papillary thyroid carcinoma had stable disease for more than 2 years.

\section{Conclusion}

Patients with varying degrees of hepatic dysfunction require appropriate dose reduction even though vorinostat pharmacokinetics are unaltered.

\section{J Clin Oncol 28:4507-4512. (c) 2010 by American Society of Clinical Oncology}

\section{INTRODUCTION}

Vorinostat is a novel anticancer agent that inhibits class I and II histone deacetylases. ${ }^{1}$ It exerts anticancer effects by direct inhibition of histone deacetylases, modulating both histone and nonhistone proteins that regulate various cell signaling pathways. ${ }^{2}$ Vorinostat induces apoptosis, inhibits proliferation, enhances anticancer immunity, and interrupts angiogenesis in a variety of preclinical cancer models. ${ }^{3-7}$ Vorinostat is approved by the US Food and Drug Administration (FDA) for the treat- ment of refractory cutaneous T-cell lymphoma. ${ }^{8}$ It is administered at a dose of $400 \mathrm{mg}$ orally daily on a continuous schedule. Vorinostat is also under evaluation in alternative treatment schedules such as twice per day administration and intermittent schedules ( 1 week on, 2 weeks off, and so on). The common toxicities associated with vorinostat include nausea, vomiting, diarrhea, fatigue, and thrombocytopenia. ${ }^{8,9}$ In addition to its activity as monotherapy in cutaneous T-cell lymphoma, vorinostat has demonstrated promising anticancer activity in combination with various cytotoxic 


\begin{tabular}{|c|c|c|c|c|}
\hline \multirow[b]{2}{*}{ Marker } & \multicolumn{4}{|c|}{ Hepatic Function Category } \\
\hline & Normal & Mild Dysfunction* & Moderate Dysfunction & Severe Dysfunction \\
\hline Total bilirubin & $\leq$ ULN & Group 1: $\leq$ ULN; group 2: $>1.0 \times$ to $1.5 \times$ ULN & $>1.5 \times$ to $3.0 \times$ ULN & $>3 \times$ ULN \\
\hline AST & $\leq U L N$ & Group 1: > ULN; group 2: any & Any & Any \\
\hline
\end{tabular}

and targeted agents. ${ }^{10-12}$ On the basis of these observations, vorinostat is being investigated in a number of solid organ and hematologic malignancies.

Vorinostat has a plasma half-life of less than 2 hours. ${ }^{13}$ The major pathways of vorinostat metabolism are $\beta$-oxidation and glucuronidation, with 4-anilino-4-oxobutanoic acid and vorinostat glucuronide being the major metabolites. Vorinostat is not a known substrate for, or inhibitor or inducer of, cytochrome P450 enzymes. Although the safety and pharmacokinetics of vorinostat have been characterized in patients with cancer with acceptable hepatic function, the pharmacokinetics and toxicity of vorinostat in patients with hepatic dysfunction have not been studied. ${ }^{9,14}$

Hepatic dysfunction, either as a result of metastases or preexisting medical conditions, occurs frequently in patients with cancer and often necessitates dose adjustments to avoid toxicity. Guidelines for appropriate use of anticancer agents in patients with hepatic dysfunction can only be developed by characterizing the safety profile of those agents in dedicated studies. Therefore, we conducted a phase I and pharmacokinetic study of vorinostat in patients with varying degrees of hepatic dysfunction.

\section{PATIENTS AND METHODS}

The primary objective of this study was to determine the maximum-tolerated dose and the dose-limiting toxicities (DLTs) of vorinostat in patients with varying degrees of hepatic dysfunction. The secondary objectives were to document the toxicities associated with vorinostat that were not dose limiting, characterize the pharmacokinetics of vorinostat, and document the anticancer activity associated with vorinostat.

\section{Inclusion Criteria}

Patients were eligible if they had histologic or cytologic confirmation of a solid organ malignancy or lymphoma for which no standard treatment options were available. For patients with hepatocellular carcinoma, an elevated $\alpha$-fetoprotein level (> $500 \mathrm{ng} / \mathrm{mL}$ ) and positive serology for hepatitis were acceptable in the absence of pathologic confirmation of diagnosis. Other inclusion criteria included the following: age more than 18 years; life expectancy of more than 3 months; Eastern Cooperative Oncology Group performance status of 0 to 2 ; absolute neutrophil count $\geq 1.5 \times 10^{9} / \mathrm{L}$; platelet count $\geq 100 \times 10^{9} / \mathrm{L}$; serum creatinine within upper limit of normal or creatinine clearance $\geq 60 \mathrm{~mL} / \mathrm{min} / 1.73 \mathrm{~m}^{2}$ for patients with elevated creatinine values; and the ability to ingest oral medications on a regular basis. Patients with biliary obstruction for which a stent had been placed were eligible, provided the stent had been in place for more than 10 days before the first dose of vorinostat and liver function had stabilized. Patients with gliomas or brain metastases who required corticosteroids or anticonvulsants were only eligible if the dose of the medication was stable and the seizure-free interval was more than 1 month. At least 3 weeks had to have elapsed since prior chemotherapy or radiotherapy before enrollment onto the study. Other exclusion criteria were as follows: major surgery within 14 days before enrollment; prior therapy with vorinostat; use of another investigational agent within 4 weeks before study entry; untreated or unstable brain metastasis; concomitant therapy with enzyme-inducing antiepileptics; uncontrolled intercurrent illness; pregnancy; and use of antiretroviral therapy for HIV. All patients provided written informed consent, and the study protocol was approved by the institutional review board at each participating institution.

\section{Study Design}

Eligible patients were stratified by the degree of hepatic dysfunction. The National Cancer Institute (NCI) Organ Dysfunction Working Group (ODWG) criteria, which are based on serum total bilirubin and serum AST concentrations, were used for categorizing hepatic dysfunction as mild, moderate, or severe (Table 1). If the AST and bilirubin values were consistent with different groups, the more abnormal parameter was used to categorize hepatic dysfunction. No distinction was made between hepatic dysfunction as a result of metastases or other causes. Up to 16 patients with normal hepatic function were to be enrolled as pharmacokinetic controls and treated with the FDAapproved dose of vorinostat of $400 \mathrm{mg}$ if they met all other eligibility criteria. There were two parts to the study. The objective of part 1 was to evaluate the pharmacokinetics of vorinostat in each of the hepatic function groups. During part 1 , all patients received a fixed, single, oral dose of $400 \mathrm{mg}$ of vorinostat after an overnight fast of more than 8 hours. This was followed by extensive peripheral blood sampling. Part 2 of the study was initiated 7 days after dosing for part 1. The primary objective of part 2 was to determine the recommended phase II dose of vorinostat for each hepatic dysfunction cohort. In part 2, vorinostat was administered on a continuous schedule as a daily, single, oral dose. Each treatment cycle consisted of 3 weeks of therapy. The starting dose of vorinostat in part 2 was selected based on the patient's degree of hepatic dysfunction. Patients were allowed to take vorinostat with food during part 2. Treatment was continued until disease progression, unacceptable toxicity resulting in more than a 2-week delay in initiating the next cycle of therapy, a decline in patient condition, or withdrawal of informed consent.

\section{Definition of $D L T$}

DLT was assessed during cycle 1 of therapy during part 2 of the study. Toxicity was graded by the NCI Common Terminology Criteria for Adverse Events (version 3.0). One or more of the following events constituted DLT: any grade 3 or 4 nonhematologic toxicity (excluding alopecia, hypersensitivity, and hepatic function abnormalities); grade 4 neutropenia lasting more than 7 days; fever associated with a neutrophil count less than $1.5 \times 10^{9} / \mathrm{L}$; grade 4 thrombocytopenia; grade 3 nausea and vomiting that occurred despite maximal antiemetic therapy; grade 3 diarrhea despite appropriate antidiarrheal therapy; toxicity-related inability to take vorinostat for at least 14 out of 21 days of therapy; and toxicity-related inability to initiate cycle 2 within 2 weeks of the scheduled start date. For patients with mild hepatic dysfunction, an increase in total bilirubin to the level defined for the severe group and lasting more than 2 weeks constituted DLT. For the moderate group, a more than 1.5 -fold increase in total bilirubin constituted DLT. A similar increase in total bilirubin for more than 2 weeks in patients with severe hepatic dysfunction constituted DLT. For patients with biliary stents, elevations of total bilirubin and AST resulting from obstructed stents were not considered as DLTs. 


\begin{tabular}{|c|c|c|c|c|}
\hline \multirow[b]{2}{*}{$\begin{array}{l}\text { Dose } \\
\text { Level }\end{array}$} & \multicolumn{4}{|c|}{ Vorinostat Dose in Hepatic Function Category $(\mathrm{mg} / \mathrm{d})$} \\
\hline & $\begin{array}{l}\text { Normal } \\
\text { Function }\end{array}$ & $\begin{array}{c}\text { Mild } \\
\text { Dysfunction }\end{array}$ & $\begin{array}{l}\text { Moderate } \\
\text { Dysfunction }\end{array}$ & $\begin{array}{c}\text { Severe } \\
\text { Dysfunction }\end{array}$ \\
\hline 1 & 400 & 300 & 200 & 100 \\
\hline 2 & - & 400 & 300 & 200 \\
\hline 3 & - & - & 400 & 300 \\
\hline 4 & - & - & - & 400 \\
\hline
\end{tabular}

\section{Dose Escalation Scheme}

All patients in the group with normal hepatic function were treated with $400 \mathrm{mg} / \mathrm{d}$. The dose-escalation schemes for patients within each hepatic dysfunction category are listed in Table 2. No dose escalation was planned beyond $400 \mathrm{mg} / \mathrm{d}$, the FDA-approved dose of vorinostat. Dose escalation proceeded simultaneously for all hepatic dysfunction categories. Patients who were not evaluable for determination of DLT were to be replaced.

\section{Patient Evaluation}

Patients underwent a complete history, physical examination, and assessment of performance status at baseline and at the initiation of each new cycle. The total bilirubin and AST were evaluated within 48 hours before initiation of therapy. A CBC with differential, serum chemistry, prothrombin time, and activated partial thromboplastin time were assessed at baseline and weekly during cycle 1 and before day 1 of each subsequent cycle. A serum pregnancy test and an ECG were performed if medically necessary. Radiographic assessment of disease status was performed after every two cycles of therapy.

\section{Dose Modifications}

Patients were required to have an absolute neutrophil count of more than $1.5 \times 10^{9} / \mathrm{L}$, platelet count of more than $100 \times 10^{9} / \mathrm{L}$, creatinine level of less than $1.5 \times$ upper limit of normal, and resolution of all other toxicity to $\leq$ grade 1 before initiation of each new cycle. During the course of the cycle, vorinostat treatment was held in the event of grade 4 neutropenia lasting more than 7 days, grade 4 thrombocytopenia, or any grade 4 nonhematologic toxicity. For grade 3 nonhematologic toxicity, treatment was withheld if the symptoms did not improve with appropriate supportive care. Under these circumstances, treatment was restarted with a reduction in vorinostat dose by $100 \mathrm{mg} / \mathrm{d}$ after resolution of the toxicity to $\leq$ grade 1 . Toxicity-related inability to start a new cycle within 2 weeks of the scheduled date resulted in removal of the patient from the study. Vorinostat dose was also reduced for subsequent cycles in the event of grade 3 or 4 neutropenia associated with fever, grade 4 neutropenia, grade 4 thrombocytopenia, uncontrolled grade 3 nausea, emesis or diarrhea, and other drug-related grade 3 or 4 nonhematologic toxicity. Dose reduction was by $100 \mathrm{mg} / \mathrm{d}$ decrements. No more than two dose reductions were allowed for a patient. Patients with severe hepatic dysfunction who experienced any of the severe toxicities described earlier were removed from study if they were being treated with the lowest dose of vorinostat $(100 \mathrm{mg} / \mathrm{d})$. If hepatic function declined to cause reclassification by more than one higher dysfunction group, the dose of vorinostat was held and restarted at the next lower dose level.

\section{Pharmacokinetic Methods}

During part 1 of the study, pharmacokinetic sampling was performed on all patients at the following time points: 30,60 , and 90 minutes before administration; and 2, 3, 4, 6, 8, 10, 12, 18, and 24 hours after administration of vorinostat. The 18-hour sampling was waived if overnight hospitalization was not feasible. At each time point, $5 \mathrm{~mL}$ of peripheral blood was collected in a red-topped Vacutainer (BD, Franklin Lakes, NJ). The blood was allowed to clot at room temperature for 30 minutes and then was centrifuged at 2,000 $\times$ $g$ for 15 minutes at $4^{\circ} \mathrm{C}$. The resulting serum was stored at $-70^{\circ} \mathrm{C}$ until assayed for vorinostat with a validated liquid chromatography-tandem mass spectrometry assay. ${ }^{13}$ Vorinostat concentration versus time data were modeled noncompartmentally using the LaGrange function as implemented by the LAGRAN computer program. ${ }^{15,16}$ The maximum serum concentration $\left(\mathrm{C}_{\max }\right)$ and the time to $\mathrm{C}_{\max }$ were determined by visual inspection of the data.

\section{Statistical Methods}

The dose-escalation rules for the study were adapted from the standard up and down $3+3$ design and maintained the basic principles of that design. The highest dose level at which less than two of six patients experienced DLT would be the recommended phase II dose for that hepatic dysfunction category. Differences in pharmacokinetic parameters across hepatic dysfunction groups were evaluated using a two-sided Kruskal-Wallis test.

\section{RESULTS}

\section{Demographics}

Fifty-seven patients were enrolled between June 2007 and July 2009 (Table 3 ). The median age was 59 years, $43 \%$ of the patients were women, and the majority of patients had an Eastern Cooperative Oncology Group performance status of 0 or 1 . The majority of patients were white. Malignancies of the GI tract were the most common tumor type. Fifteen patients were enrolled onto the normal hepatic function control group.

\section{Toxicity}

The most common DLT was grade 4 thrombocytopenia, which accounted for eight of the nine DLTs. Of the first six patients with mild hepatic dysfunction treated with $300 \mathrm{mg} / \mathrm{d}$, one experienced a DLT

\begin{tabular}{|c|c|}
\hline Characteristic & No. of Patients ( $N=57$ ) \\
\hline \multicolumn{2}{|l|}{ Age, years } \\
\hline Median & 59 \\
\hline Range & $25-81$ \\
\hline Female & 24 \\
\hline \multicolumn{2}{|l|}{ ECOG performance status } \\
\hline 0 & 9 \\
\hline 1 & 40 \\
\hline 2 & 8 \\
\hline \multicolumn{2}{|l|}{ Primary cancer } \\
\hline Adenoid cystic carcinoma & 5 \\
\hline Breast cancer & 2 \\
\hline Cholangiocarcinoma & 4 \\
\hline Colon cancer & 21 \\
\hline Hepatocellular carcinoma & 4 \\
\hline Lung cancer & 3 \\
\hline Pancreatic cancer & 4 \\
\hline Rectal cancer & 6 \\
\hline Thymic cancer & 3 \\
\hline Other & 5 \\
\hline \multicolumn{2}{|l|}{ Hepatic dysfunction category } \\
\hline Normal & 15 \\
\hline Mild & 16 \\
\hline Moderate & 15 \\
\hline Severe & 11 \\
\hline \multicolumn{2}{|l|}{ Race/ethnicity } \\
\hline White & 54 \\
\hline African American & 1 \\
\hline Asian & 2 \\
\hline
\end{tabular}

Abbreviation: ECOG, Eastern Cooperative Oncology Group. 


\begin{tabular}{|lccccc}
\hline \multicolumn{5}{c}{ Table 4. Toxicity } \\
\hline \multirow{5}{*}{ Toxicity } & Grade 1 & Grade 2 & Grade 3 & Grade 4 & All Grades \\
\cline { 2 - 6 } & 0 & 5 & 0 & 0 & 5 \\
\hline Anemia & 3 & 5 & 0 & 0 & 8 \\
Anorexia & 0 & 0 & 3 & 0 & 3 \\
Dehydration & 1 & 4 & 1 & 0 & 6 \\
Diarrhea & 1 & 3 & 2 & 1 & 7 \\
Fatigue & 0 & 0 & 2 & 2 & 4 \\
Hyperbilirubinemia & 1 & 2 & 0 & 0 & 3 \\
Leukopenia & 0 & 1 & 3 & 0 & 4 \\
Lymphopenia & 3 & 1 & 0 & 0 & 4 \\
Nausea & 0 & 2 & 1 & 0 & 3 \\
Neutropenia & 2 & 4 & 5 & 8 & 19 \\
Thrombocytopenia & 2 & & & \\
\hline NOTEyyyy
\end{tabular}

NOTE. Toxicity represents the worst grade for each patient that was possibly, probably, or definitely related to vorinostat and occurred in more than $5 \%$ of the patients during the entire course of treatment.

(grade 4 thrombocytopenia). At the next dose level of $400 \mathrm{mg} / \mathrm{d}$, one of the first five patients with mild hepatic dysfunction experienced grade 4 thrombocytopenia. The last patient enrolled onto this cohort was incorrectly dosed at $300 \mathrm{mg} / \mathrm{d}$ as a result of an error and experienced a DLT (grade 3 diarrhea with dehydration). This resulted in a total of two DLTs in the seven patients treated at the $300 \mathrm{mg} / \mathrm{d}$ dose level. Considering this with the episodes of non-dose-limiting thrombocytopenia noted at $400 \mathrm{mg} / \mathrm{d}$, the decision was made not to treat additional patients at $400 \mathrm{mg} / \mathrm{d}$. Therefore, $300 \mathrm{mg} / \mathrm{d}$ was determined to be the recommended phase II dose for patients with mild hepatic dysfunction. One of six evaluable patients with moderate hepatic dysfunction who were treated with $200 \mathrm{mg} / \mathrm{d}$ had a DLT, and two of four patients with moderate hepatic dysfunction who were treated with $300 \mathrm{mg} / \mathrm{d}$ had a DLT. Two evaluable patients with severe dysfunction who were treated with $200 \mathrm{mg} / \mathrm{d}$ experienced a DLT, and one of six patients with severe hepatic dysfunction who were treated with $100 \mathrm{mg} / \mathrm{d}$ experienced a DLT. As is common in studies of patients with hepatic dysfunction, a number of patients enrolled onto the study (mild, $\mathrm{n}=4$; moderate, $\mathrm{n}=5$; severe, $\mathrm{n}=5$ ) were not evaluable for DLT because they could not complete the first cycle of therapy as a result of a decline in their overall disease status or because of other disease-related complications. Treatmentrelated non-dose-limiting toxicities included anorexia, nausea, diarrhea, fatigue, and neutropenia (Table 4). There were no treatment-related deaths. In general, the toxicity profile was consistent with the known safety profile of vorinostat.

\section{Pharmacokinetics}

There was large interpatient variability in pharmacokinetic parameters in each hepatic dysfunction group (Table 5). The values for vorinostat $\mathrm{C}_{\max }$, time to $\mathrm{C}_{\max }$, half-life, area under the curve and clearance observed in this study were compatible with values reported in previous studies of oral vorinostat. ${ }^{9,10,17,18}$ There were no statistically significant differences in any pharmacokinetic parameter across hepatic dysfunction groups. Moreover, the patients experiencing DLTs in each hepatic group were not those with the highest vorinostat area under the curve or $\mathrm{C}_{\max }$ or longest half-life and were not the patients who entered the study with the lowest pretreatment platelet counts.

\section{Efficacy}

Twelve patients experienced stable disease. Three of these patients had colon cancer, and one patient each had ovarian carcinoma, thyroid carcinoma, melanoma, breast cancer, and thymoma. The stable disease of the patient with papillary thyroid carcinoma has lasted more than 2 years. A patient with adenoid cystic carcinoma of the salivary gland had a partial response that was associated with a marked improvement in performance status. This led to the enrollment of four additional patients with adenoid cystic carcinoma, the majority of whom experienced prolonged disease stabilization $(4,12$, 12 , and 13 months). Three patients with adenoid cystic carcinoma and the patient with thyroid carcinoma are still on therapy with vorinostat. Notably, all the patients with adenoid cystic carcinoma and the patient with thyroid carcinoma had experienced disease progression on the previous regimens before entry onto the study.

\section{DISCUSSION}

Our study demonstrates that the doses of vorinostat used in patients with hepatic dysfunction should be lower than the dose approved for patients with normal hepatic function. There were no unusual or previously undescribed vorinostat-associated toxicities in patients with hepatic dysfunction. This study was conducted by the NCIODWG, which has previously conducted studies of a number of anticancer agents in patients with hepatic dysfunction. ${ }^{19-22}$ The study used a design whereby all patients initially received a single, fixed dose of vorinostat regardless of the degree of hepatic dysfunction, which allowed for optimal comparison of the pharmacokinetics of vorinostat across all hepatic dysfunction categories. This design was developed by Miller et $\mathrm{al}^{23}$ in their phase I study of sorafenib in patients with hepatic dysfunction. Despite there being no statistically significant differences in vorinostat pharmacokinetic parameters among patients with varying degrees of hepatic dysfunction, the maximum-tolerated dose of

\begin{tabular}{|c|c|c|c|c|c|c|c|c|c|c|c|}
\hline \multirow{2}{*}{$\begin{array}{l}\text { Hepatic Function } \\
\text { Group }\end{array}$} & \multirow{2}{*}{$\begin{array}{c}\text { No. of } \\
\text { Patients }\end{array}$} & \multicolumn{2}{|c|}{$\mathrm{C}_{\max }(\mu \mathrm{M})$} & \multicolumn{2}{|c|}{$t_{\max }$ (hours) } & \multicolumn{2}{|c|}{$\mathrm{t}_{1 / 2}$ (hours) } & \multicolumn{2}{|c|}{$\mathrm{AUC}_{\mathbb{I N F}}(\mu \mathrm{M} \cdot \mathrm{h})$} & \multicolumn{2}{|c|}{$\mathrm{Cl}_{\mathrm{APP}}(\mathrm{L} / \mathrm{min})$} \\
\hline & & Mean & SD & Mean & SD & Mean & SD & Mean & SD & Mean & SD \\
\hline Normal & 15 & 1.4 & 0.5 & 1.4 & 0.8 & 2.5 & 1.2 & 5.1 & 1.9 & 6.4 & 5.1 \\
\hline Mild dysfunction & 15 & 2.2 & 1.1 & 2.3 & 2.0 & 2.0 & 1.4 & 7.7 & 3.4 & 4.0 & 1.8 \\
\hline Moderate dysfunction & 15 & 1.7 & 0.6 & 2.7 & 1.9 & 3.5 & 2.8 & 7.5 & 2.4 & 3.8 & 1.6 \\
\hline Severe dysfunction & 9 & 1.8 & 0.7 & 2.6 & 2.2 & 2.9 & 1.5 & 8.3 & 5.1 & 4.2 & 2.3 \\
\hline
\end{tabular}

Abbreviations: $C_{\text {max }}$ maximum vorinostat concentration in serum; $t_{\max }$, time at which $C_{\max }$ occurred; $t_{1 / 2}$, half-life; $A U C_{I N F}$, area under the serum vorinostat concentration versus time curve from 0 to infinity; $\mathrm{Cl}_{\mathrm{APP}}$, apparent vorinostat clearance, defined as dose/area under the curve; $\mathrm{SD}$, standard deviation. 
vorinostat decreased progressively as hepatic dysfunction worsened. This could reflect variation in treatment tolerance due to overall patient condition that is related to hepatic function. Alternatively, the toxicity could be due to vorinostat metabolites that were not measured in this study but that might accumulate in patients with decreased hepatic metabolic and excretory capacity. Unfortunately, vorinostat metabolites could not be quantitated because authentic standards and stable-labeled internal standards were not available. Another observation in our study involves the inability of many patients to complete even one cycle of therapy, thus rendering them nonevaluable for assessment of DLT. This is a common problem in studies of patients with hepatic dysfunction because in the absence of effective therapy, a rapid decline in patient condition often occurs. In the NCI-ODWG phase I study of imatinib in patients with hepatic dysfunction, nearly $50 \%$ of patients were nonevaluable due to rapid deterioration of their clinical condition. ${ }^{20}$

Our study also noted promising anticancer activity in a variety of malignancies, which is consistent with prior reports of single-agent vorinostat in patients with head and neck cancer, breast cancer, and non-small-cell lung cancer. ${ }^{24-26}$ Of the five patients with adenoid cystic carcinoma enrolled, one had a partial response, and four had stable disease. Given the fact that adenoid cystic carcinoma is a chemotherapy-refractory disease in which responses are not often seen, a phase II study to evaluate the activity of vorinostat in patients with adenoid cystic carcinoma is being planned.

In summary, the inability to deliver the recommended dose of vorinostat to patients with hepatic dysfunction despite the absence of altered pharmacokinetics disposition underscores the importance of conducting phase I studies of novel agent in patients with organ dysfunction, even if no obvious changes in drug handling explain the clinical data that demonstrate the need for dose modifications.

\section{AUTHORS' DISCLOSURES OF POTENTIAL CONFLICTS} OF INTEREST

Although all authors completed the disclosure declaration, the following author(s) indicated a financial or other interest that is relevant to the subject matter under consideration in this article. Certain relationships marked with a " $U$ " are those for which no compensation was received; those relationships marked with a "C" were compensated. For a detailed description of the disclosure categories, or for more information about ASCO's conflict of interest policy, please refer to the Author Disclosure Declaration and the Disclosures of Potential Conflicts of Interest section in Information for Contributors.

Employment or Leadership Position: None Consultant or Advisory Role: Suresh S. Ramalingam, Merck (C); Chandra P. Belani, Merck (C) Stock Ownership: None Honoraria: None Research Funding: Patricia LoRusso, Merck; Jan H. Beumer, Merck; Merrill J. Egorin, Merck Expert Testimony: None Other Remuneration: None

\section{AUTHOR CONTRIBUTIONS}

Conception and design: Suresh S. Ramalingam, Shivaani Kummar, S. Percy Ivy, Chandra P. Belani, Merrill J. Egorin

Administrative support: Suresh S. Ramalingam, Mara Yerk, Chandra P. Belani, Merrill J. Egorin

Provision of study materials or patients: Suresh S. Ramalingam, Shivaani Kummar, John Sarantopoulos, Stephen Shibata, Patricia LoRusso, R. Donald Harvey, Julianne Holleran, Jan H. Beumer, S. Percy Ivy, Chandra P. Belani, Merrill J. Egorin

Collection and assembly of data: Suresh S. Ramalingam, Shivaani Kummar, John Sarantopoulos, Stephen Shibata, Patricia LoRusso, R. Donald Harvey, Mara Yerk, Yan Lin, Jan H. Beumer, S. Percy Ivy, Chandra P. Belani, Merrill J. Egorin

Data analysis and interpretation: Suresh S. Ramalingam, Shivaani Kummar, John Sarantopoulos, Stephen Shibata, Patricia LoRusso, R. Donald Harvey, Julianne Holleran, Yan Lin, Jan H. Beumer, S. Percy Ivy, Chandra P. Belani, Merrill J. Egorin

Manuscript writing: Suresh S. Ramalingam, Shivaani Kummar, John Sarantopoulos, Stephen Shibata, Patricia LoRusso, R. Donald Harvey, Yan Lin, Jan H. Beumer, S. Percy Ivy, Chandra P. Belani,

Merrill J. Egorin

Final approval of manuscript: Suresh S. Ramalingam, Shivaani Kummar, John Sarantopoulos, Stephen Shibata, Patricia LoRusso, R. Donald Harvey, Mara Yerk, Julianne Holleran, Yan Lin, Jan H. Beumer, S. Percy Ivy, Chandra P. Belani, Merrill J. Egorin

\section{REFERENCES}

1. Dokmanovic M, Clarke C, Marks PA: Histone deacetylase inhibitors: Overview and perspectives. Mol Cancer Res 5:981-989, 2007

2. Marks PA, Richon VM, Breslow $R$, et al: Histone deacetylase inhibitors as new cancer drugs. Curr Opin Oncol 13:477-483, 2001

3. Neuzil J, Swettenham E, Gellert N: Sensitization of mesothelioma to TRAIL apoptosis by inhibition of histone deacetylase: Role of $\mathrm{BCl}-\mathrm{xL}$ downregulation. Biochem Biophys Res Commun 314: 186-191, 2004

4. Marks P, Rifkind RA, Richon VM, et al: Histone deacetylases and cancer: Causes and therapies. Nat Rev Cancer 1:194-202, 2001

5. Richon VM, Webb Y, Merger R, et al: Second generation hybrid polar compounds are potent inducers of transformed cell differentiation. Proc Natl Acad Sci U S A 93:5705-5708, 1996

6. Yu C, Rahmani M, Almenara J, et al: Histone deacetylase inhibitors promote STI571-mediated apoptosis in STI571-sensitive and -resistant Bcr/Abl+ human myeloid leukemia cells. Cancer Res 63:21182126, 2003

7. Kim MS, Kwon HJ, Lee YM, et al: Histone deacetylases induce angiogenesis by negative regulation of tumor suppressor genes. Nat Med 7:437443, 2001

8. Duvic M, Talpur R, Ni X, et al: Phase 2 trial of oral vorinostat (suberoylanilide hydroxamic acid, SAHA) for refractory cutaneous T-cell Iymphoma (CTCL). Blood 109:31-39, 2007

9. Kelly WK, O'Connor OA, Krug LM, et al: Phase I study of an oral histone deacetylase inhibitor, suberoylanilide hydroxamic acid, in patients with advanced cancer. J Clin Oncol 23:3923-3931, 2005

10. Ramalingam SS, Parise RA, Ramanathan RK, et al: Phase I and pharmacokinetic study of vorinostat, a histone deacetylase inhibitor, in combination with carboplatin and paclitaxel for advanced solid malignancies. Clin Cancer Res 13:3605-3610, 2007

11. Witta SE, Gemmill RM, Hirsch FR, et al: Restoring E-cadherin expression increases sensitivity to epidermal growth factor receptor inhibitors in lung cancer cell lines. Cancer Res 66:944-950, 2006

12. Fakih MG, Pendyala L, Fetterly G, et al: A phase I, pharmacokinetic and pharmacodynamic study on vori- nostat in combination with 5-fluorouracil, leucovorin, and oxaliplatin in patients with refractory colorectal cancer. Clin Cancer Res 15:3189-3195, 2009

13. Parise RA, Holleran JL, Beumer JH, et al: A liquid chromatography-electrospray ionization tandem mass spectrometric assay for quantitation of the histone deacetylase inhibitor, vorinostat (suberoylanilide hydroxamicacid, SAHA), and its metabolites in human serum. J Chromatogr B Analyt Technol Biomed Life Sci 840:108-115, 2006

14. Kelly WK, Richon VM, O'Connor O, et al: Phase I clinical trial of histone deacetylase inhibitor: Suberoylanilide hydroxamic acid administered intravenously. Clin Cancer Res 9:3578-3588, 2003

15. Yeh KC, Kwan KC: A comparison of numerical integrating algorithms by trapezoidal, LaGrange, and spline approximation. J Pharmacokinet Biopharm 6:79-98, 1978

16. Rocci ML Jr, Jusko WJ: LAGRAN program for area and moments in pharmacokinetic analysis. Comput Programs Biomed 16:203-216, 1983

17. Fujiwara $Y$, Yamamoto $N$, Yamada $Y$, et al: Phase I and pharmacokinetic study of vorinostat (suberoylanilide hydroxamic acid) in Japanese 
patients with solid tumors. Cancer Sci 100:17281734, 2009

18. Rubin EH, Agrawal NG, Friedman EJ, et al: A study to determine the effects of food and multiple dosing on the pharmacokinetics of vorinostat given orally to patients with advanced cancer. Clin Cancer Res 12:7039-7045, 2006

19. Gibbons J, Egorin MJ, Ramanathan RK, et al: Phase I and pharmacokinetic study of imatinib mesylate in patients with advanced malignancies and varying degrees of renal dysfunction: A study by the National Cancer Institute Organ Dysfunction Working Group. J Clin Oncol 26:570-576, 2008

20. Ramanathan RK, Egorin MJ, Takimoto $\mathrm{CH}$, et al: Phase I and pharmacokinetic study of imatinib mesylate in patients with advanced malignancies and varying degrees of liver dysfunction: A study by the National Cancer Institute Organ Dysfunction Working Group. J Clin Oncol 26:563-569, 2008

21. Takimoto $\mathrm{CH}$, Graham MA, Lockwood G, et al: Oxaliplatin pharmacokinetics and pharmacodynamics in adult cancer patients with impaired renal function. Clin Cancer Res 13:4832-4839, 2007

22. Mulkerin D, Remick SC, Ramananthan RK, et al: A dose-escalating and pharmacologic study of bortezomib in adult cancer patients with impaired renal function. J Clin Oncol 24:87s, 2006 (suppl; abstr 2032)

23. Miller AA, Murry DJ, Owzar K, et al: Phase I and pharmacokinetic study of sorafenib in patients with hepatic or renal dysfunction: CALGB 60301. J Clin Oncol 27:1800-1805, 2009
24. Blumenschein GR Jr, Kies MS, Papadimitrakopoulou VA, et al: Phase II trial of the histone deacetylase inhibitor vorinostat (Zolinza, suberoylanilide hydroxamic acid, SAHA) in patients with recurrent and/or metastatic head and neck cancer. Invest New Drugs 26:81-87, 2008

25. Luu TH, Morgan RJ, Leong L, et al: A phase II trial of vorinostat (suberoylanilide hydroxamic acid) in metastatic breast cancer: A California Cancer Consortium study. Clin Cancer Res 14:7138-7142, 2008

26. Traynor AM, Dubey S, Eickhoff JC, et al: Vorinostat (NSC\# 701852) in patients with relapsed non-small cell lung cancer: A Wisconsin Oncology Network phase II study. J Thorac Oncol 4:522-526, 2009

\section{Genitourinary Cancers Symposium: Register Today!}

This pre-eminent genitourinary (GU) oncology event (February 17-19, 2011, Orlando, Florida) is your best opportunity to learn about the latest strategies in prevention, screening, evaluation, and management of GU cancers. This Symposium offers multidisciplinary educational sessions and oral abstract and poster presentations on urothelial carcinoma, and prostate, renal, penile, and testicular cancers.

New this year:

- Best of Journals Session open to all registered attendees-no ticket required

- Increased time for Q\&A

- Abstract Supplement in Journal of Clinical Oncology

- Virtual Meeting included as part of registration fee

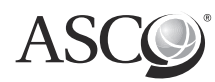

American Society of Clinical Oncology 